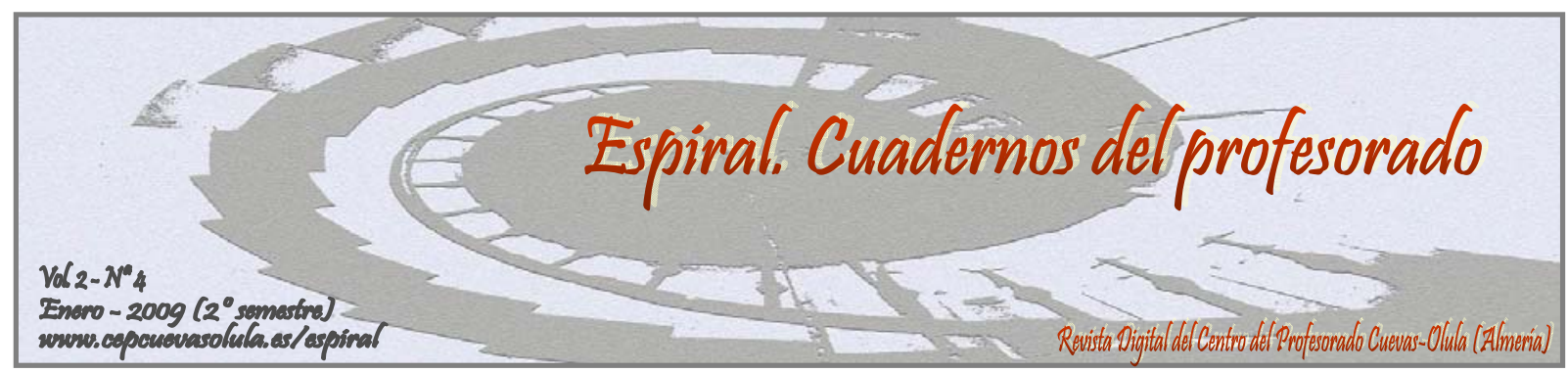

\title{
EL BALONCESTO COMO CONTENIDO CURRICULAR EN EDUCACIÓN SECUNDARIA. PROPUESTA PARA SU ENSEÑANZA BAJO UN MODELO COMPRENSIVO
}

\section{BASKETBALL AS PART OF THE CURRICULUM IN SECONDARY EDUCATION. MOTION FOR EDUCATION: A COMPREHENSIVE MODEL}

\author{
María Cañadas Alonso ${ }^{(1)}$, Javier García Rubio ${ }^{(2)}$, Isabel Parejo González ${ }^{(2)}$ \\ ${ }^{(1)}$ Facultad de Ciencias del Deporte. Universidad de Murcia

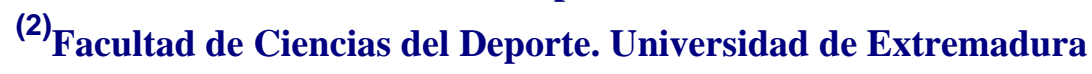

RESUMEN: Este documento pretende mostrar una forma de entender la enseñanza del baloncesto. Partiendo de los objetivos de la Educación Física en secundaria y de la estructura de un deporte colectivo como es este deporte, proponemos la enseñanza del baloncesto basado en la comprensión del propio juego, con el objeto de conseguir alumnos activos, que se impliquen cognitivamente durante las sesiones. Dejando a un lado las propuestas que desde una perspectiva teórica a menudo resultan poco prácticas para los profesores, queremos proporcionar ideas y principios a tener en cuenta útiles a la hora del diseño de las actividades de cada una de las sesiones que conformaran la unidad didáctica para la enseñanza de este deporte.

Palabras clave: baloncesto, educación física, toma de decisión, metodología alternativa, modelo comprensivo.

ABSTRACT: This document aims to show a way of understanding the teaching of basketball. Based on the objectives of Physical Education in Secondary Education and the structure of a collective sport like it is this one, we propose the teaching of basketball based on the understanding of the game, with the goal of getting active students, who engage cognitively during the different sessions. Leaving to a side, the proposes which are often impractical for the teachers, from a theorical perspective, we want to provide ideas and principles to be considered in the design of activities of each session that conform the didactic unit for the teaching of this sport.

Key words: basketball, physical education, decision-making, alternative approach, understanding model.

Cañadas Alonso, M., García Rubio, J. \& Parejo González, I. (2009). El baloncesto como contenido curricular en educación secundaria. Propuesta para su enseñanza bajo un modelo comprensivo. Espiral. Cuadernos del Profesorado [en línea], 2(4), 66-76. Disponible en: http://www.cepcuevasolula.es/espiral.

Fecha de recepción: 18/12/2008

Fecha de aceptación: 02/04/2009
Enviar correspondencia a: mariacanadas@um.es 


\section{1.- INTRODUCCIÓN.}

Los deportes colectivos de cooperaciónoposición, como el baloncesto, están constituidos por habilidades predominantemente perceptivas, abiertas y de regulación externa. Siendo así, es necesario un procesamiento de la información del entorno con objeto de percibir, evaluar, anticiparse y adaptarse a los constantes cambios que se suceden en el juego (Méndez, 1998). El desarrollo del baloncesto en un entorno en constante cambio exige de la toma de decisiones en un corto espacio de tiempo (Tavares, 1997).

La práctica de deportes colectivos supone un medio favorecedor, siempre y cuando esté bien orientado, para el desarrollo del jugador en el ámbito psicomotor, cognitivo y socioafectivo (Mesquita, 1997).

La enseñanza de este deporte en las clases de Educación Física puede ser abordada desde dos metodologías. Un modelo más tradicional y mecanicista, basado en la enseñanza de gestos técnicos descontextualizados del deporte en sí. El punto central de este modelo es el trabajo de la técnica mediante la repetición del modelo mecánico. Por el contrario, la metodología alternativa, basada en un modelo comprensivo, resalta la importancia de la toma de decisiones y los aspectos tácticos del juego (Mitchell, Oslin \& Griffin, 2003). El "Teaching Games for Understanding" (TGfU) referente de este modelo, se basa en la comprensión del deporte y propone el aprendizaje mediante juegos modificados o reducidos que mantienen la misma estructura y dinámica que el juego global (Griffin, Mitchell \& Oslin, 1997).

La enseñanza tradicional de los deportes está basada en el desarrollo de habilidades aisladas y de su práctica en situación descontextualizada. En la enseñanza bajo el T.G.f.U., son trabajadas cada una de las partes del deporte adquiriendo significado en la globalidad del mismo. La principal diferencia entre las dos metodologías es que, mientras el objetivo principal de la metodología tradicional o mecanicista es desarrollar jugadores habilidosos, el objetivo de la alternativa es que los alumnos comprendan y entiendan el deporte (Butler, 1998). Los alumnos deben ser capaces de comprender e interpretar lo que pasa en las sesiones de Educación Física y no ser simples espectadores. La enseñanza de un deporte como el baloncesto planteada desde una perspectiva alternativa se caracteriza por un aprendizaje significativo, el profesor como orientador del proceso y un alumnado activo, principios de una enseñanza constructivista.

La metodología alternativa permite a los estudiantes saber cómo, cuándo y porqué es adecuado hacer cada una de las acciones. Con este tipo de práctica los alumnos conocen cada una de las habilidades del deporte y cuándo es mejor aplicarlas en la situación de juego (Griffin et al., 1997). Una de las principales contribuciones que hace esta metodología es mejorar los movimientos y el posicionamiento de los jugadores sin balón en el campo, así como las acciones de apoyo al jugador con balón en ataque o las de ayuda en defensa (Rezende, 2004). En los deportes abiertos hay una constante interacción ataque-defensa y las demandas del juego cambian constantemente. La selección de la respuesta o toma de decisión correcta es crucial para solucionar problemas que se dan en la situación de juego (Iglesias, Moreno, Santos, Cervelló \& Villar, 2005). La toma de decisión adecuada se relaciona con la cantidad de conocimiento almacenado en la memoria, así como con la efectiva y eficiente ejecución de los movimientos adecuados (Williams, Ward \& Chapman, 2003).

Se ha demostrado que los alumnos que aprenden un deporte a través de esta metodología alternativa toman mejores decisiones que aquellos que son enseñados a través de una metodología tradicional (Holt, Etrean \& Bengoechea, 2002). Así mismo, la enseñanza basada en el aprendizaje de la toma de decisiones tácticas favorece la transferencia a otros deportes con principios similares (Mitchell \& Oslin, 1999). Esto genera un conocimiento transversal y común a los deportes incluidos en el curriculum educativo, que facilita el aprendizaje de los deportes a lo largo del ciclo formativo.

\section{2.- PRINCIPIOS A TENER EN CUENTA AL ELABORAR LAS SESIONES PARA LA ENSEÑANZA DEL BALONCESTO EN EDUCACIÓN FÍSICA.}

En este apartado se proporciona una serie de pautas que pueden resultar de utilidad para el diseño de cada una de las tareas que conforman la unidad didáctica de este deporte, así como orientaciones para la actuación del profesor durante la sesión. Todo ello con el objeto de, atendiendo a la estructura de este deporte y principios del aprendizaje, crear un entorno de práctica adecuado para el alumnado. 


\section{Progresar de lo simple a lo complejo.}

En base a este principio se plantea la organización de las tareas progresando de situaciones más simples hasta situaciones más complejas.

En el caso concreto de los deportes colectivos, la incertidumbre de esta actividad procede de las acciones de oponentes y compañeros. La presencia de compañeros y oponentes supone un número de estímulos a los que atender, así como posibles opciones para la toma de decisiones (Ruiz \& Sánchez, 1997). Por ello, al principio se proponen situaciones reducidas en las que el número de compañeros y oponentes, y consecuentemente los puntos en los que centrar la atención, se ve disminuido facilitando la atención en los puntos que se refieren a la técnica de la habilidad que aún no se domina o que se quiere trabajar en ese momento. Progresivamente se va incluyendo mayor número de estímulos al ir introduciendo compañeros y oponentes. También el número de opciones o posibles soluciones a cada una de las decisiones que tiene que ir tomando el jugador se ven incrementadas con la presencia de compañeros y adversarios. Incrementar y reducir el número de oponentes y compañeros permite manipular la dificultad perceptiva y decisional de las tareas facilitando el aprendizaje del alumno.

La secuenciación de propuestas en la sesión puede iniciarse con situaciones de 1vs1, pasando por situaciones de $2 \mathrm{vs} 1,2 \mathrm{vs} 2,3 \mathrm{vs} 2$, 3vs3, hasta llegar a la situación más cercana al juego real y también más compleja, 4vs4 ó 5vs5.

Generar desequilibrios en las tareas para el trabajo de cada una de las fases de juego.

Para facilitar el aprendizaje de una habilidad bien de la fase de ataque o de defensa, podemos trabajar en la situación más favorecedora. Se sabe que la acción del oponente es un agente que limita y dificulta la acción del individuo, tanto en ataque como en defensa. Por ello, en las tareas de enseñanza se pueden crear desequilibrios a favor de la fase de juego en la que se centra el aprendizaje.

Un ejemplo que ayuda a explicar a qué hace referencia este principio sería el hecho de que para facilitar las acciones ofensivas se limita la acción de la defensa prohibiendo el robo del balón, limitando sus movimientos, reduciendo el número de oponentes, etc.

\section{Trabajar con oposición.}

El aprendizaje de una habilidad en una situación contextualizada favorece su transferencia al juego real. El baloncesto es un juego de oposición en el que el jugador realiza las acciones frente a un oponente, en muchas de las ocasiones como reacción a la acción de este. Por este motivo, es recomendable que siempre que sea posible la práctica se desarrolle en esta situación, con oposición.

La presencia del oponente no puede ser un obstáculo que retrase en exceso el aprendizaje del jugador. Se puede modular la complejidad de la tarea limitando la acción del defensor, progresando desde un comportamiento pasivo (sólo molesta, puede incluso permanecer estático), semi-activo (movimiento de brazos, sin desplazamiento), hasta uno más activo (se desplaza y mueve brazos, pero no puede robar balón $\mathrm{o}$, finalmente, movimiento libre, permitiéndole robar balón).

\section{Trabajo conjunto de táctica y técnica.}

Los contenidos de un deporte como el baloncesto puedes clasificarse en gestos técnico-tácticos y conductas táctico-técnicas, es decir la técnica y la táctica de un deporte. El trabajo conjunto de ambas dimensiones del juego favorece la contextualización del aprendizaje ya que durante el juego las acciones técnicas y tácticas se suceden de forma simultánea.

La propuesta de actividades basadas en juegos modificados o reducidos del juego real es un medio que propicia el aprendizaje de una habilidad desde el punto de vista táctico y técnico. A través de este tipo de tareas se pretende que el alumno conozca el qué, cómo y cuándo de la acción, es decir, por un lado aprende su utilidad táctica (cuándo y cómo es más adecuado utilizarlo), y por el otro lado cómo se ejecuta la respuesta motora (el qué). El profesor puede corregir la acción táctica y la acción técnica del alumno en una misma propuesta, además de que éste conoce de forma conjunta las implicaciones técnicas y tácticas de la habilidad. Sin embargo, esto no significa que no puedan utilizarse situaciones más analíticas en las que trabajar de forma específica cada uno de estos aspectos de la habilidad. En el caso de que alguna de las acciones presente demasiada dificultad puede aislarse y trabajar en ejercicios analíticos en los que la repetición del gesto de forma independiente permite fijar la atención en los 
aspectos que no se dominan. Aún así, siempre es recomendable tras este proceso, aplicar lo aprendido en una situación global.

\section{Utilizar feedback interrogativo.}

Tal y como se ha comentado anteriormente uno de los objetivos de estas sesiones debe ser el desarrollo de la dimensión cognitiva del alumno. El tipo de tareas que se plantean bajo esta perspectiva se basan en presentar un problema al alumno, el cual debe resolver él mismo buscando las posibles soluciones. El profesor guiará su acción planteándole preguntas que le hagan reflexionar sobre qué es más adecuado hacer. De este modo, el profesor no le proporciona la solución directamente, sino que le surtirá de las herramientas que le permitan encontrar la solución por sí mismo. De esta forma, el alumno es un sujeto activo que participa en la sesión y que comprende por él mismo qué es lo que debe hacer en cada situación favoreciendo un aprendizaje más significativo. Esta concepción de la enseñanza se relaciona con el modelo comprensivo según el cual el alumno debe participar en situaciones que precisen elección e iniciativa (Nuviala \& Tamayo, 2003).

\section{Propuestas lúdicas que supongan un reto.}

Este principio hace referencia al tipo de tareas que pueden plantearse en la sesión. Se propone la utilización del juego como medio para la enseñanza. El juego fomenta la motivación de los alumnos propiciando un entorno favorecedor para el aprendizaje. Los juegos en grupo, en los que se plantean situaciones de cooperación y oposición, además de un resultado socializador, desarrollan el trabajo coordinativo entre los participantes, el cuál será útil para un deporte colectivo como el baloncesto. En estas edades, las propuestas que suponen un reto o la superación de un obstáculo benefician la implicación del alumno en la realización de las tareas. Para ello, se pueden utilizar premisas como premiar cuando se realicen ciertas acciones, plantear un objetivo o una puntuación a conseguir, etc. Por otro lado, la organización de torneos, ligas internas, competiciones, etc., puede ser un medio que propicie el aprendizaje. La competición, con un planteamiento educativo, es una situación en la que se puede aplicar todo lo aprendido en las clases y seguir desarrollándolo. También supone un medio de evaluación en el cual el alumno aprecia qué aspectos domina y cuáles debe mejorar.

\section{Potenciar aquello que se quiere trabajar.}

Siguiendo la línea de lo expuesto anteriormente, si se quiere mantener el interés del alumnado en la sesión se deben plantear premisas, objetivos, sistemas de puntuación, etc., que favorezcan la motivación del alumno hacia los contenidos y tareas de enseñanza. Asociar una puntuación a la realización de una acción propicia que esta misma se desarrolle y, por lo tanto, que el alumno quiera practicarla más.

A modo de ejemplo para el aprendizaje del bloqueo del rebote se puede establecer la norma de que la persona que consiga un rebote gane un punto.

\section{3.- PROPUESTAS PRÁCTICAS.}

Se pasa a plantear los aspectos más relevantes a tratar en cada una de las partes de la sesión. Estas partes tendrán un comienzo y final delimitado claramente, y se tratarán todas con la misma importancia para que así lo perciba el alumno. Tan importante es la acción, cómo la reflexión sobre la misma.

\section{INTRODUCCIÓN.}

La sesión se iniciará explicando cuál es el contenido a tratar. Se pueden plantear preguntas a los alumnos sobre la utilidad de los aspectos a trabajar, sin olvidar que es recomendable que los alumnos lo descubran y lo conozcan en la práctica. No se deben proporcionar las recetas $o$ soluciones a los problemas que serán planteados en la sesión.

\section{CALENTAMIENTO.}

Juego de calentamiento. La sesión puede iniciarse con un juego de calentamiento, más o menos especifico y continuar con la movilidad y estiramiento específico de este deporte. Como ejemplo de juego de calentamiento puede proponerse el juego de los 10 pases. La actividad plantea dos equipos, uno de ellos debe intentar conseguir el objetivo a través del que se obtiene punto (meter el balón en portería, en una canasta, en unos aros, etc.) pero antes deben conseguir 10 pases entre los componentes de su equipo. El otro equipo debe evitarlo interceptando el balón.

Mediante esta propuesta pueden comenzar a trabajarse los contenidos de la sesión. Esta actividad se utiliza como ejercicio introductorio al desarrollo de la sesión. No es solamente un calentamiento físico, sino también mental que prepara al cuerpo y a la mente para la clase. 
Tabla 1. Variables, índices y puntuaciones de rendimiento del GPAI (Oslin, Mitchell \& Griffin, 1998).

\begin{tabular}{|c|c|}
\hline Implicación en el juego $=$ & $\begin{array}{l}\mathrm{N}^{\circ} \text { total de respuestas apropiadas }+\mathrm{n}^{\circ} \text { de veces que una habilidad esta } \\
\text { bien ejecutada }+\mathrm{n}^{\circ} \text { de veces que una habilidad está mal ejecutada }+\mathrm{n}^{\circ} \text { de } \\
\text { decisiones no apropiadas }\end{array}$ \\
\hline $\begin{array}{l}\text { Índice de toma de decisión } \\
\text { (ITD) }=\end{array}$ & $\mathrm{N}^{\mathrm{o}}$ de decisiones apropiadas $/ \mathrm{n}^{\mathrm{o}}$ de decisiones no apropiadas. \\
\hline $\begin{array}{l}\text { Índice de ejecución de habilidad } \\
(\text { IEH })=\end{array}$ & $\mathrm{N}^{\mathrm{o}}$ de ejecuciones eficaces $/ \mathrm{n}^{\mathrm{o}}$ de ejecuciones no eficaces \\
\hline Índice de apoyo(IA) = & $\begin{array}{l}\mathrm{N}^{\mathrm{o}} \text { de desplazamientos de apoyo apropiados } / \mathrm{n}^{\circ} \text { de desplazamientos de } \\
\text { apoyo no apropiados }\end{array}$ \\
\hline Puntuación de rendimiento & $\mathrm{ITD}+\mathrm{IEH}+\mathrm{IA} / 3$ \\
\hline
\end{tabular}

Tras la realización de juego pueden plantearse preguntas a los alumnos introduciendo el contenido a trabajar. Como ejemplo para trabajar la toma de decisión, formular preguntas como: ¿A quién pasar?, ¿Avanzar con el balón? ¿Por qué? ¿Qué hacer tras pasar? Para el movimiento de recepción: ¿Cómo me muevo para recibir el balón? O para trabajar la ocupación de espacios libres (O.E.L.): ¿Correr todos hacia el balón? ¿Ocupar los espacios dónde están mis compañeros o dónde no hay nadie?

\section{PARTE PRINCIPAL.}

Juegos en progresión. En cada sesión se planteara una progresión, basada en los criterios establecidos anteriormente para la adecuación del aprendizaje. A continuación se presentará un ejemplo de propuesta para el trabajo de los tres contenidos.

\section{PARTE FINAL.}

Juego de aplicación. Esta parte la constituye el juego final en el que se aplica todo lo aprendido en esta sesión y en las anteriores. Supone la situación más compleja de la sesión y la más global.

A continuación, con la intención de seguir profundizando en las propuestas prácticas para cada una de las sesiones de esta unidad didáctica, se proporcionan una serie de tareas que, de modo secuencial, pueden ser planteadas para trabajar tres contenidos básico de este deporte como son: la toma de decisión en el juego de $1 \times 1$, el movimiento de recepción y la ocupación de espacios libres (O.E.L). A través de la sesión se va a intentar relacionar los objetivos propuestos con los contenidos, metodología, etc.., todo concretado en las tareas, que se organizarán de acuerdo al tiempo, espacio o material disponible (Nuviala \& Tamayo, 2003).

Como primer paso para el inicio del juego en el baloncesto se trabajará la toma de decisión del alumno con balón.

Se parte desde la situación de juego más básica en la que puede encontrarse el jugador con balón, 1vs1. La toma de decisión es uno de los contenidos que supone la formación básica del jugador con balón en el juego individual.

El movimiento de recepción es la acción que realizan dos jugadores para ponerse en comunicación motriz, intercambiándose el balón, en la que se producen un pase y una recepción de forma consciente.

En ocasiones estos han sido considerados como gestos técnico-tácticos individuales, pero en realidad son colectivos ya que ponen en relación a dos jugadores. Supone el principal medio grupal que pone en comunicación a los jugadores.

La ocupación de espacios libres supone el sistema de iniciación al juego colectivo en la fase de ataque. La primera forma de trabajar el juego colectivo en la iniciación es con este medio, que es la distribución armónica, equilibrada y eficaz de los jugadores por el terreno de juego y su evolución por los distintos puestos específicos.

En esta evolución los desplazamientos no están predeterminados, pero hay una serie de reglas básicas para que este desplazamiento no sea rígido pero tampoco caótico. 


\begin{tabular}{|c|c|c|c|c|}
\hline \multicolumn{5}{|c|}{ CONTENIDO: TOMA DE DECISION (1X1). } \\
\hline 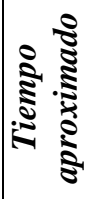 & $\begin{array}{l}\text { REPRESENTACIÓN } \\
\text { GRÁFICA }\end{array}$ & DESCRIPCIÓN DE LA ACTIVIDAD & CONTENIDO & 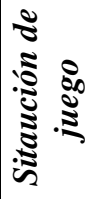 \\
\hline $10^{\prime}$ & & $\begin{array}{l}\text { Jugar 1x1. El balón comienza en el } \\
\text { atacante. } \\
\text { Si hay distancia con el defensor lanzo, } \\
\text { si no hay distancia boto. } \\
\text { Obligatorio atacar por el lado débil. } 3 \\
\text { botes máximo. } \\
\text { Juego en los } 5 \text { espacios de exteriores. } \\
\text { Después de cada ataque rotar a la } \\
\text { siguiente posición. }\end{array}$ & $\begin{array}{l}\text { Toma de } \\
\text { decisión: } \\
\text { Lanzar o } \\
\text { botar } \\
\text { (penetrar) }\end{array}$ & $1 \times 1$ \\
\hline $10^{\prime}$ & & $\begin{array}{l}\text { 1x1. Si hay distancia con el defensor } \\
\text { lanzo, si no hay distancia boto o paso. } \\
\text { Obligatorio atacar por lado débil. } 3 \\
\text { botes máximo. } \\
\text { Juego en los } 5 \text { espacios de exteriores. } \\
\text { Después de cada ataque rotar a } \\
\text { siguiente posición. }\end{array}$ & $\begin{array}{l}\text { Toma de } \\
\text { decisión: } \\
\text { Lanzar } \\
\text { Botar } \\
\text { (penetrar) } \\
\text { Pasar }\end{array}$ & $2 \times 2$ \\
\hline $10^{\prime}$ & & $\begin{array}{l}\text { 1x1. Si hay distancia con el defensor } \\
\text { lanzo, si no o boto o paso a cualquiera } \\
\text { de mis compañeros. } \\
\text { Obligatorio atacar por lado débil. } 3 \\
\text { botes máximo. } \\
\text { Juego en los } 5 \text { espacios de exteriores. } \\
\begin{array}{l}\text { Después de cada ataque rotar a } \\
\text { siguiente posición. }\end{array}\end{array}$ & $\begin{array}{l}\text { Toma de } \\
\text { decisión: } \\
\text { Lanzar } \\
\text { Botar } \\
\text { (penetrar) } \\
\text { Pasar (2 } \\
\text { opciones de } \\
\text { pase) }\end{array}$ & $3 \times 2$ \\
\hline $10^{\prime}$ & & $\begin{array}{l}\text { Si hay distancia con el defensor lanzo, } \\
\text { si no o boto o paso a cualquiera de mis } \\
\text { compañeros. } \\
\text { Obligatorio atacar por lado débil. } 3 \\
\text { botes máximo. } \\
\text { Juego en los } 5 \text { espacios de exteriores. } \\
\text { Después de cada ataque rotar a } \\
\text { siguiente posición. }\end{array}$ & $\begin{array}{l}\text { Toma de } \\
\text { decisión: } \\
\text { Lanzar } \\
\text { botar } \\
\text { (penetrar) } \\
\text { Pasar (2 } \\
\text { opciones de } \\
\text { pase o más) }\end{array}$ & $\begin{array}{c}3 \times 3 \\
0 \\
4 \times 4\end{array}$ \\
\hline
\end{tabular}

\section{4.- LA EVALUACIÓN DEL APRENDIZAJE EN BALONCESTO.}

Como parte integrante de la unidad didáctica el proceso de evaluación se encarga de comprobar si los objetivos planteados al inicio de la unidad han sido alcanzados. En el caso concreto de los contenidos específicos de este deporte a nivel procedimental, si el alumno ha adquirido las destrezas y habilidades que le permitan desarrollar el juego del baloncesto. La evaluación debe seguir la misma perspectiva que la adoptada a la hora de plantear la enseñanza de este deporte, lo que significa que debe evaluar aquellos aspectos a los que se ha dado prioridad y de la forma en que han sido trabajados. 
El modelo que se ha presentado en este documento está enfocado desde una perspectiva comprensiva y, por lo tanto, la evaluación que se plantea pretende no evaluar únicamente el desarrollo técnico y motriz del alumno sino también su comprensión del juego.

Tradicionalmente, la evaluación de las acciones de juego se ha desarrollado a través de pruebas mecánicas, test de habilidades, relegando a un papel secundario la comprensión del juego. La mayoría de los instrumentos de evaluación están planteados desde un enfoque tradicional y mecanicista.

La propuesta que se presenta en este documento recomienda la evaluación directa del rendimiento técnico-táctico durante el juego, utilizando instrumentos de observación directa y registros del desempeño deportivo.

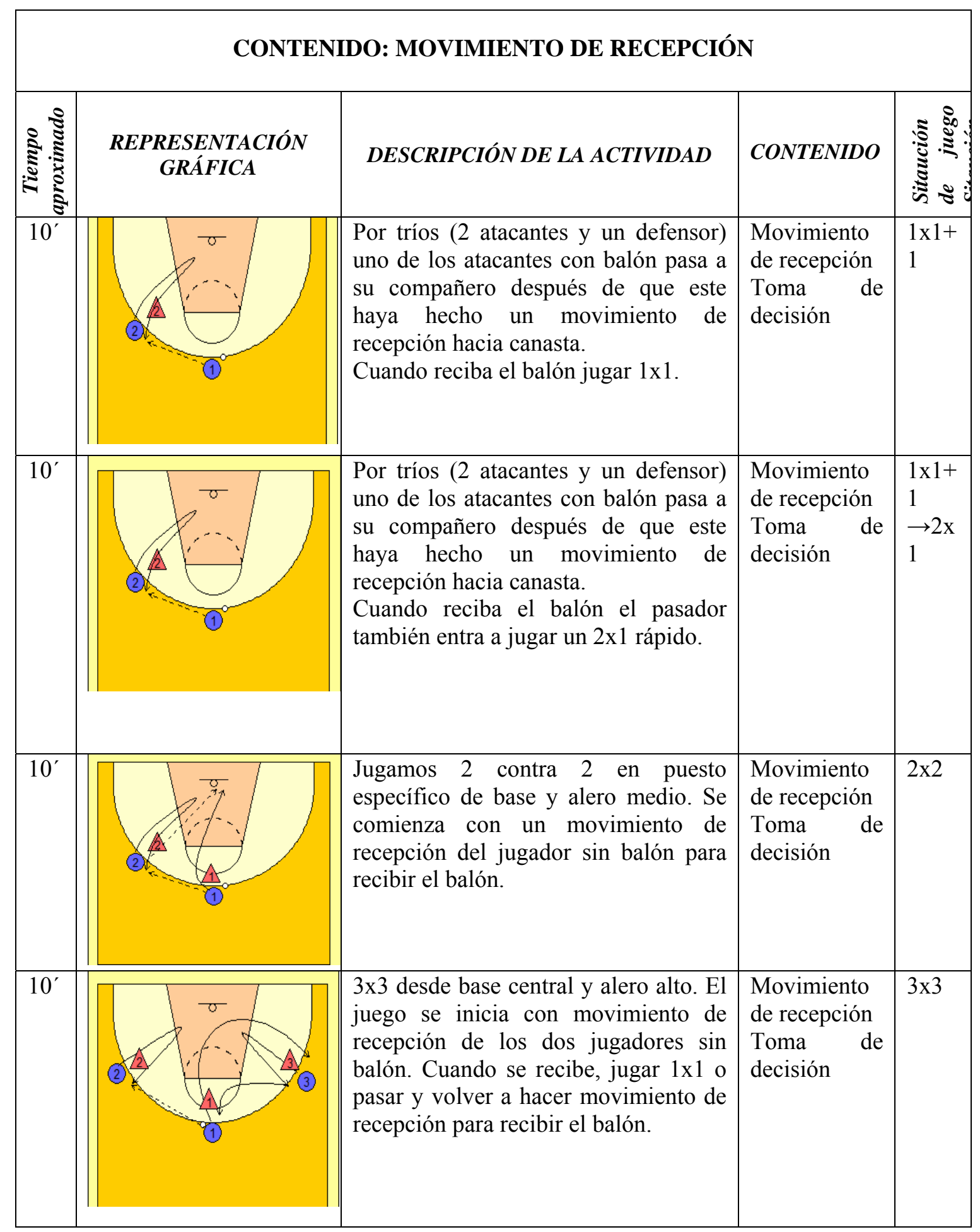


Los instrumentos actualmente utilizados (scouts) deben ser revisados con el objeto de incluir el análisis cualitativo de los aspectos tácticos así como las acciones sin posesión del balón, las grandes olvidadas (Rezende, 2004).

García y Cañadas (2005), realizan una adaptación del "Game Performance Assessement Instrument" G.P.A.I de Mitchell y Oslin (1999), para evaluar el aprendizaje de jugadores en categoría minibasket (10-11 años). Este instrumento de evaluación consiste en una serie de ítems que miden la capacidad táctica de los jugadores, no sólo en las acciones con balón, sino también en las acciones sin balón, que ocupan el $90 \%$ del tiempo de juego (tabla 1 ).

Esta idea de evaluación puede ser incorporada por el profesorado al proceso de evaluación con el objeto de evaluar la capacidad cognitiva del alumno, en este caso, en el desempeño durante el juego del baloncesto.

Los criterios de evaluación de esta propuesta serían:

1. Que el alumno sea capaz de aplicar las habilidades específicas adquiridas a si-

\section{CONTENIDO: DESPLAZAMIENTOS POR EL CAMPO/ OCUPACIÓN DE ESPACIOS LIBRES.}

\begin{tabular}{|c|c|c|c|c|}
\hline 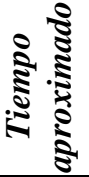 & $\begin{array}{c}\text { REPRESENTACIÓN } \\
\text { GRÁFICA }\end{array}$ & DESCRIPCIÓN DE LA ACTIVIDAD & CONTENIDO & : \\
\hline $10^{\prime}$ & & $\begin{array}{l}3 \text { espacios/ } 2 \text { jugadores. El alumno } \\
\text { con balón decide lo que va a hacer } \\
\text { (botar o pasar) y en función de esto se } \\
\text { mueve por el espacio. Solo se puede ir } \\
\text { al espacio libre. } \\
\text { Variante 1: ir al espacio ocupado. } \\
\text { Variante 2: pasar siempre por debajo } \\
\text { del aro antes de ir a ocupar un sitio } \\
\text { libre u ocupado. }\end{array}$ & $\begin{array}{l}\text { Ocupación } \\
\text { de espacios } \\
\text { libres }\end{array}$ & $2 \times 0$ \\
\hline $10^{\prime}$ & & $\begin{array}{l}4 \text { espacios/ } 3 \text { jugadores. El alumno } \\
\text { con balón decide lo que va a hacer } \\
\text { (botar o pasar) y en función de esto se } \\
\text { mueve por el espacio. Los demás } \\
\text { alumnos se mueven en función de } \\
\text { esto. Solo se puede ir al espacio libre. } \\
\text { Variante: ir al espacio ocupado. }\end{array}$ & $\begin{array}{l}\text { Ocupación } \\
\text { de espacios } \\
\text { libres }\end{array}$ & $3 \times 0$ \\
\hline $10^{\prime}$ & & $\begin{array}{l}\text { Repetir ejercicios } 1 \text { y } 2 \text { pero con } \\
\text { defensores. } \\
\text { Defensa en inferioridad numérica. Si } \\
\text { hay opción de pase, pasar el balón y } \\
\text { jugar } 1 x 1 \text {. }\end{array}$ & $\begin{array}{l}\text { Ocupación } \\
\text { de espacios } \\
\text { libres }\end{array}$ & $\begin{array}{l}2 \times 1 \\
3 \times 2\end{array}$ \\
\hline $10^{\prime}$ & & $\begin{array}{l}3 \times 3 \text { en posiciones de base central y } \\
\text { alero alto. Después de pasar ocupar el } \\
\text { espacio libre. Jugar } 1 \times 1 \text { cuando sea } \\
\text { posible. }\end{array}$ & $\begin{array}{l}\text { O.E.L. } \\
\text { Toma de } \\
\text { decisión }\end{array}$ & $3 \times 3$ \\
\hline
\end{tabular}


tuaciones reducidas del juego, prestando una atención especial a los elementos perceptivos y de ejecución.

2. Que el alumno resuelva problemas de decisión planteados por la realización de tareas motrices deportivas utilizando habilidades específicas y evaluando la adecuación de la ejecución al objetivo previsto.

3. Que coordine las acciones propias con las del equipo interpretando con eficacia la táctica para lograr la cohesión y eficacia cooperativas (por ejemplo en situación de $3 \times 3$ ).

La prueba de evaluación consiste en el desarrollo de situaciones de juego a través de las cuales será evaluada la acción del alumno. Pueden proponerse situaciones individuales (1vs1), grupales (2vs2, 3vs3) ó colectivas (4vs4, $5 \mathrm{vs} 5)$, en base al tipo de contenidos que se quiera evaluar, en las que el alumno deba resolver las situaciones que se le presentan, resultado del propio juego.

El profesor mediante observación directa irá registrando las acciones-decisiones adoptadas por el alumno evaluado. Anteriormente, deben haber sido establecidos los criterios que determinan que una acción/decisión es errónea o acertada.

Mediante este sistema de evaluación se puede evaluar de forma conjunta la técnica y la táctica, y sobre todo de forma contextualizada partiendo de la estructura y la lógica del propio deporte. De este modo, se concede la importancia que merece la implicación y desarrollo cognitivo del alumnado mediante un deporte colectivo como el baloncesto.

Se precisa de la creación y sobre todo de la utilización de instrumentos de evaluación que midan la capacidad del alumno adquirida para el desempeño en el juego del baloncesto. Esto incluye la evaluación de los aspectos tácticos y técnicos que han sido enseñados, y que permiten el posterior desarrollo del juego.

\section{5.- CONCLUSIONES.}

Las tareas propuestas para el aprendizaje de las habilidades propias de los deportes colectivos deben asegurar la creación de un bagaje motor, que permita dotar al alumno de una serie de técnicas que le faciliten participar en el deporte con éxito. En el caso de los deportes colectivos, además de este bagaje, se requiere de una implicación cognitiva por parte del deportista.
La presencia de oponentes y compañeros genera una serie de situaciones cambiantes en las que el jugador debe encontrar y aplicar la solución adecuada a la circunstancia, todo esto bajo un proceso reflexivo, mediante el cual el jugador percibe el entorno, toma la decisión adecuada y ejecuta la respuesta. Este esquema debe ser aplicado y practicado en las sesiones de aprendizaje en Educación Física, lo que nos asegura que el aprendizaje es fruto de la reflexión y no de la memorización y automatización sin cognición.

En cuanto a la dimensión social, el hecho de tratarse de deportes de equipo lleva implícito la relación con otros. Por tanto, las clases suponen un medio de transmisión de valores como: honestidad, tolerancia, solidaridad, trabajo en equipo, respeto, etc.

Pero estos valores no sólo contribuyen a la formación como persona del alumno, sino que la práctica en situaciones que propicien la cooperación genera un clima positivo que favorece las relaciones interpersonales, lo que facilita el desarrollo de las habilidades y, consecuentemente, genera el aumento del aprendizaje que solo se consigue con el trabajo en conjunto.

La utilización de una metodología alternativa, basada en un modelo comprensivo, que propicia el aprendizaje significativo, el desarrollo integral del alumno y la participación activa del mismo, supone el medio más adecuado para enseñar un deporte como el baloncesto en las clases de Educación Física.

\section{6.- REFERENCIAS BIBLIOGRÁFICAS.}

Butler, J. (1998). Factory or village green? Two approaches to teaching games education. Education for Life. AIESEP proceedings (pp. 171-179). Garden City: Adelphi University.

García, J. \& Cañadas, M. (2005). Evaluación de la capacidad táctica de un equipo de minibasket. En R. Martínez de Santos, S. J. Ibáñez \& L. M. Sautu (Coords.), Actas del III Congreso Ibérico de Baloncesto (pp. 7686). Vitoria-Gasteiz: AVAFIEP.

Griffin, L., Mitchell, S. \& Oslin, J. (1997). Teaching sport concepts and skills: A tactical games approach. Champaign, IL: Human Kinetics.

Holt, N.L., Strean, W.B. \& Bengoechea, E.G. (2002). Expanding the teaching games for understanding model: new avenues for future 
research and practice. Journal of Teaching in physical education, 21, 162-176.

Iglesias, D., Moreno, M. P., Santos, F. J., Cervelló, E. M. \& Villar, F. (2005). Cognitive expertise in sport: relation between procedural knowledge, experience and performance in youth basketball. Journal of Human Movement Studies, 49(1), 65-76.

Méndez, A. (1998). La observación in vivo del rendimiento deportivo, un instrumento de análisis en iniciación al baloncesto. Lecturas: Educación Física y deportes. Revista digital, 12. Recuperado el 14 de mayo de 2005 de http://www.efdeportes.com/

Mesquita, I. (1997). Propuesta metodológica. En A. Graca \& J. Oliveira. La enseñanza de los juegos deportivos (pp.157-200). Barcelona: Paidotribo.

Mitchell, S. \& Oslin, J. (1999). Assessment in game teaching. Physical Education \& Sport Pedagogy, 4(2), $162-172$.

Mitchell, S. A., Oslin, J. L. \& Griffin, L. L. (2003). Sport foundations for Elementary Physical Education. A tactical games approach. Champaign, IL: Human Kinetics.

Nuviala, A. \& Tamayo, J. (2003). Las tareas motrices en la enseñanza de los elementos técnico-tácticos de los deportes. Retos. Nuevas tendencias en Educación Física, Deporte y Recreación, 4, 5-10.
Oslin, J, Mitchell, S. A. \& Griffin, L. L. (1998). The game performance assessment instrument: development and preliminary validation. Journal of Teaching in Physical Education, 17, 231-243.

Rezende, A. (2004). Métodos de estudio de las habilidades tácticas (3): inventario de habilidades deportivas. Lecturas de Educación Física y deportes. Revista Digital, 70. Recuperado el 15 de mayo de 2005

de http://www.efdeportes.com/efd70/tatica.htm

Ruiz, L. M. \& Sánchez Bañuelos, F. (1997). Rendimiento deportivo: claves para la optimización de los aprendizajes. Madrid: Gymnos.

Tavares, F. (1997). El proceso de la información en los juegos deportivos. En A. Graca \& J. Oliveira, La enseñanza de los juegos deportivos (pp. 35-48). Barcelona: Paidotribo.

Williams, A. M., Ward, P. \& Chapman, C. (2003). Training perceptual skill in field hockey: is there transfer from the laboratory to the field? Research quarterly for exercise and sport, 74, 98-103.

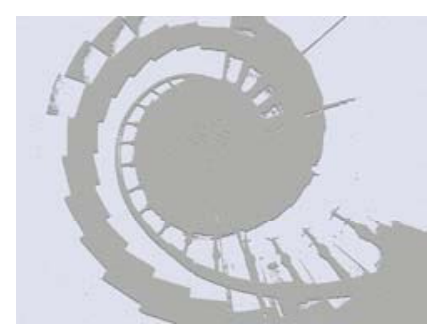

